\title{
[ 282$]$
}

II.

BEOBACHTUNGEN

is $b \in r$

die tägliche Veründerung in der Abweichung der Magnetnadel und Unterfuchungen über deren Urfache,

$\nabla \mathbf{o r}$

$$
\begin{aligned}
& \text { Profeflor H íLLSTRö M } \\
& \text { i n } \AA \text { b o. *) }
\end{aligned}
$$

- Die Phyfiker ftimmen in Folgendem überein : i. dafs die Magnetnadel täglich zwei Schwingungen, eine gegen Weften, die andere gegen Often macht; 2. dafs ihre Abweichung ungefähr um 8 Uhr Morgens am geringften, und um I oder 2 Uhr Nachmittags am grölsten ift; 3. dafs die Abweichung nach Weiten während diefer Zeit wächft, von 1 oder 2 Uhr Nachmittags an aber bis Abends um 8 oder 9 Uhr abnimmt; und dals 4 . das Nordlicht in diefen regelmäfsigen Schwingungen befonclere Störungen bewirkt. Endlich foll 5. nach Caffini der menfchliche Körper auf die Magnetnadel durch Abftofsung wirken.

*) Ausgezogen von Herrn Adjunct Droy?en in Greifswalde, aus einer Disfertatio de variationibus declinationis magneticae diurnis, 'rraefide Guft. Gabr. Hälljtrom, Refp. Snellmann. Aboae 1803. 


\section{[ $28 j]$}

Wir machten unfre Magnetuadel von einer gebrauchten Uhrfeler, und hingen fie in ihrem Schwerpunlite an einem Faden von Menlchenhaaren auf. Die Abweichung wurde durch ein Mikrofkop aus 2 Convexgläfern, welches die Gegenftäncle verkehrt darftellte, beobachtet. An diefem MikroSkop war ein vorzüglich gearbeitetes Mikrometer von Dollond angebracht, deffen fefter horizontaler Faden in den magnetifchen Meridian geftellt wurde, und deffen beweglicher Faden fich durch eine Schraube über dem Nordpole der Magnetnallel fo verfchieben liefs, dals man clic Veränderungen der Abweichung genau meffen konnte, inclem die Schraube durch einen Zeiger die Zahl der Lindrehungen und Vierzigftel einer Umdrehung angab. Der Faden, welcher die Magnetnadel trugr, war fo an einem, meffingenen Haken aufigehängt, dafs keine Drehung deffelben Statt fand, und clas Mikrofkop wurde auf Holz fo geftellt, dafs cter Norclpol der Nadel deutlich gefehen werden konnte. Da Luftftröme die Nadel in immerwährender Bewegang erhielten, mufsten wir fie in ein hölzernes Gefäfs voll Waffer hängen, und nun erft liefsen fich an ihr die Veränderungen der Abweichung bequerr bemerken.

$\mathrm{Um}$ zu beftimmen, wie grofs der Abweichungs winkel fey, der einer Schraubenumdrehung de Mikrometers entfpricht, Itellten wir das Mikrofko] in einiger Entfernung über einen fchwedifchen gec metrifchen Maalsftab, fo dafs die Abtheilunge 


\section{[ $28 ;]$}

des Maafsitabes deutlich zu fehen waren, und fuchten, um wie viel Theile a auf der Scale, der Faden des Mikrometers bei $b$ Undrchungen der Schraube fortzurücken fchien. Hiernach mufste die Länge des Bogens, wclchen der.Pol der Nadel durchlief, wenn er um eine Schraubenumdrehung vor oder zurück ging, $=\frac{a}{b}$ feyn. Setzt man allo die halbe Länge der Nadel vom $A$ ufhängepunkte an $=c$, ferner den Winkel, defien Bogen dem Radius gleich ift, $=e$, und endlich den Schwingungswinkel, der mit einar Schraubenuindrehung des Nikrometers überein ftimmte, $=x:$ fo war $c: \frac{a}{b}=e: x$ und $x=\frac{a e}{b c}$. Es ift alfo bei $m$ Undrehungen des Schraubenmikrometers, der diefen Úmdrehungen correfpondirenrle Schwingungsivinkel $=\frac{a e^{m}}{b c}$. In der erften Reihe der Beobachtungen war $a=0, I$ geomet. Zoll; $\iota^{\circ}=7,75, c=2,27$ und $e=206264,8^{\prime \prime}$, alfo der Schwingungswinkel für $m$ Schraubenumdrehungen, $=m \cdot 1 \times 72^{\prime \prime}=m 2 \cdot\left(19^{\prime} 32^{\prime \prime}\right)$.

Es war nicht unfre Abiicht, die wah́re Abwejchung der Magnetuadel zu beobachten, *) fondern nur die tägliche Veründerung derfelben zu beftimmen. Wir. bedienten uns daher ohne weiteres diefer Gleichung bei der Recluction unfrer Beobachtungen,

*) Diefe hatten wir an einer andern Nadel von 7 Zoll Länge, welche $1^{\prime}$ Veränderung zeigte, wegen der Reibung aber nicht beweglich genug war, uin die raglichen Veränderungen anzugeben, ungefähr $9^{\circ} 30^{\prime}$ wefllich gefunden.

H. 


\section{[ 285 ]}

indem es uns genug war, dafs das grö̈fsere $m$ eine gröfsere Declination anzeigte. Alles Eifen legten wir forgfältig von uns, und was fich an Eifen im Mikrometer oder fonft wo, mehr oder minder von der Nadel entfernt, befand, behiclt immer die nämliche Lage gegen fie. Die Beobachtungen gefchahen im Anfange des Septembers I So3.

Die Beobachtungen der erften 4 Tage gaben eine grofse tägliche Veränderung in der Abweichung; auch fchieneu diefe Veränclerungen ein ganz anderes Gefetz zu befolgen, als andere gefunden haben. Denn am 2ten, 4 ten und 5 ten September nalm die weftliche Declination von 8 oder 9 Ühr Morgens bis I, 2 oder 3 Uhr Nachmittags ab, von diefer Zeit an aber bis 9 oter ro Uir Abends zu, und am 3ten Sept. wurde fie faft den ganzen Tag lang imnuer grölser. *)

Diefe uns unerwartete Befchaffenheit der Veränderungen in der Abweichung glaubten wir nun senauer erforfchon zu mäflen. Wir nahmen wielerun diefelbe Nadel, nachiem wir ihr fo viel magnetifche Kraft, als fie fallen konnte, ertheilt hatten, gaben ihr aher jetzt eine folche Einrichtung, Jafs fich an ihr die Variationen der $\Lambda$ bweichung in loppelter Gröfse, als zuvor, zeigen mulsten. Wir

*) Herr Hall ftröm theilt diefe Beohachtungen in einer Tabelle anit, die ich jedoch, fo wic die fol. gende Tabelle, weglaffe, da die liefultate hier die Hauptlache find.

Dr. 


\section{[ 2866$]$}

klebten nämlich mit Wachs den Südpol der Magnetnadel an eine platt gedrickte Bleikugel, von $\frac{1}{2}$ Zoll Durchneffer, und an diefe fenkrecht eine meffingene Angel, vermittelit deren die Kugel an die Kette von Menfchenhaaren gehängt wurde, und fo hing, dafs die Nadel horizontal fchwebte. Da wir glaubten, des Luftftromes wegen; die Nadel ins Waffer tauchen zu mülen, fo überzogen wir auch fie mit Wachs, damit fie nicht vom Rofte leiden möchte. Das Waffer war in einem thönernen Gefäfse, und clie Variationen der Abweichung wurclen mit dem vorigen Mikrofkope und Mikrometer beobachtet. $a, b$ und $c$ behielten ihse vorigen Werthe, nur war jetzt $c=4,4$ Zoll, wefshalb der Veränderungswinkel bei einer JUmusehung der Mikrometerfchraube jetzt Io' $5^{\prime \prime}$ betrug.

Die Beobachtungen, welche nun angeftellt wurden, ftimmen daril überein, dafs die weftliche $\mathrm{Ab}$ weichung um $x, 2,3$ oder 4 Uhr Nachmittags am gröfsten, an Morgen abèr und am Abent am geringften ift, wie man das auch bei Peobachtungen an andern Orten gefunden hat. Den waliren Grund, warum diefe Refultate von den vorigen fo bedeutend abıveichen, künnen wir, aus Mangel dahin gehöriger Verfuche, noch nicht angeben. Beide Reihen von Verfuchén wurden mit gleicher V'orficht angeftellt, nur dafs die Magnetnadel am 2ten, 4 ten und 5 ten September fich dem Sonnenlichte aurgeietzt befand, indels dieies an den anderin Tagen nicht der Fall war. Am zten und I gten Sept. war nünlich der 


\section{[ 287 ]}

Himmel mit Wolken bedeckt, am 2often Sept. und am 4 ten, 5 ten, 6ten und 7 ten October, an welchen Tagen wir unfre zweite Reihe von Beobachtungen anftellten, welche mit dem gewöhnlichen Gefetze überein ftimmten, war der Himmel zwar klar, die Magnetnadel ftand aber nicht im Sonnenlichte.

Ein Abftofsen zwilchen der Magnetnadel und dèm menfchlichen Körper, deffen Caffini erwähnt, konnten wir nicht bemerken; cloch find wir geneigt, zu glauben, dafs die Gegenwart vieler Perfonen die weftliche Abweichung der Magnetnadel fchwächt. Denn ofters traf es fich, dafs die Nadel dem Erclmerician näher kan, wenn fich mehrere Menfchen der einen oder andern Seite näherten. Sonnenfchein und die Gegenwart mehrerer Perfonen brachten alfo gleiche liirkung hervor. Wir wïden fonach diefe Wirkungen aus der vermehrten Wärme der Nadel herleiten, wäre die Abweichungsnadel von Luft, und nicht von Waffer umgeben gewefen. Ob aber in Waffer die Nadel fo fchmell habe erwärmt werden können, dafs dadurch binnen $15^{\prime}$, während die Temperatur des Waffers fo wenig erhöhet wurde, eine bemerkliche Veränderung der Abweichung hatte bewirkt und beobachtet werclen künuen; daran müllen wir zweifeln. *)

*) Konnten etwa Luftftröme, durch partiale Verände. rungen der Teinperatur hewirkt, den Faden, woran die Nadel hing, ein wenig aus feiner Lago brin. 


\section{[ 288 ]}

Die Verfuche Canton's zeigen, dals die anziehende Kraft der Magnetnadel durch Erwärmung verändert-werde; ob eine ähnliche Wirkung in Beziehung auf die Abweichung'Statt findet, vordiente durch wiederhohlte Verfuche ausgemittelt $\mathrm{zu}$ werclen.

Auch vom Nordlichte wird nach unfrer.Erfahrung die weftliche Abweichung vermindert; ob aber beftändig oder zufällig, ift noch nicht aušsgemacht.

In einer zweiten Disfertation *) prufft Hr. Prof. Ha llftrö dì Theorieen über die tüglichen Veranderungen dër Abrveichung. Ich hebe aus ihr das Neue und Eigenthümliche aus.

I. Mairan**) fchreibt dicfe Veräncierungen dem Ausftrümen der Sonnenatmofphäre zu, welche auf cler. Ercle die magnetifche Richtung ftöre; und diefes fchliefst er daraus, weil beim Nordlichte, weiches er aus der Vermifchung der Sonnenund Exdatmofphäre crklïrt, die Ofcillationen der

Nadel

gen? Doch das ift kaum glauhlich, da die Beobachter, wie fie angeben, die Vorficht gebraucht hatten, die Nadel fo aufzuhüngen, dals der Faden auf fie keine Drellkraft ausübte. d. $H$.

*) Animadverfiones ciria hypothejes ad explicandas acus magneticae variationes diurnas excrgitatas. Fraer fide Hüllftrïm, refir. Wahlften. Abone 1803 .

**) Traité phyfique et hifitorique de l'Aurore boreale, Paris 1759, p. 45. 


\section{$[289]$}

Nidel merklicher find. - Hieraus aber wardo folgen, dats die Veränderangen der Declination grölser und wenigger regelmälsig in der Zeit feyn mülstea, wenn die Yordlichter häufiger fond; jeno fird aber gerack im Sonmer am bemerkbarften. Aucis vingle daraus folgen, dafs immer Nordlichter feyn'muisten, da jene Veränderungen bleibend find.

2. Canton*) beobachtete, dafs eine Magnetnadel, die von zwei Magneten in einer beliebigen Richtung gehalten wird, fich dem einen nähere, wein der andere erwärnt wird, und zu diefem zurück gehe, wenn er wieder erkaltet. Hieraus gtaubte er auf die Urfache' der Veränderung der Abrveichung fchliefsen zu können, weil die Erde zuerft in Often ervairmt werde.

Prevoft fcheint dieferr noch am erften beizuftimmen, doch glaubt er Canton's Meinung aus dem Grunde verwerfen zu muiffen, weil in tiefen Bergwerken keine Veränderung in der Erwärmung durch die Strahlen der Sonne zu bemerken ley. Er fucht daher vielmehr den Grund diefer Veränderungen in der Atmofphäre und auf der Erdoberfäche. $\left.{ }^{* *}\right)$

Gegen $\boldsymbol{E}$ anton's Hypothefe wendet Coulomb ein, die magnetifche Kraft der Erde müffe täglich abnehmen und fchnell verfchwinden, wenn

*) Philojoph. Transactions, 1759, p. 401. ה.

**) De l'origine des forces magnétiques. Généve 1788. Anual. d. Phyfil. B. 19. St. 3. J. 1805. St, 3. 


\section{[ 290 ]}

die Sonnenwärme fre verminderte, *) wefswegen er fie von der Lage der Erde und Sonne gegen einandèr ableiten zu müffen glaubt. Nach feiner Meinnung ift die Sonnenatmofphäre felbft eine magnetifche-Fluffigkeit, und wirkt auf die magnetifche

*) Coulomb würde gefunden baben, dafs diefe Einwendung gegen Canton gar keine Kraft bat, wenn er deffen Verfuche, wie wir es gethan haben, wiederhohlt hätte. Wir fanden hierbei, dals die magnetifche anziehende und abftofsende Kraft um fo mehr abnimmt, je wärmer der Magnet wird, und dafs fie, wenn er erkaltet, wieder zunimmt. Wir fetzten den Nordpol eines künfflichen Magnets in einem Abftande ron 2 Fufs rom magnetifchen Meridian einer frei aufgehăngten Nadel, dem Nordpole derfelben gegen über an der Offeite, damit fie etwas vom Magnete abgeftofsen würde, und beob. achteten ihre Abweichung bei der Temperatur der fie umgebenden Luft, $\left(+20^{\circ}\right.$ Celfius.) Darauf gof. fen wir heifses Walfer auf den Mlagnet, und be. merkten, dafs bei $+80^{\circ}$ Temperatur des Magnets die Declination der Nadel um einen Winkel von $2^{\prime} 46^{\prime \prime}$ geringer war. Als wir umgekehrt den Magnet in das Waffer durch Schnee bis $0^{\circ}$ erkälteten, wrichs die Declination wieder um einen Winkel von $3^{\prime} 42^{\prime \prime}$, welches für jeden Grad des Celfius fchen Therrnometers einen Winkel von $2^{\prime \prime}, 8$ beträgt. Wir Rellten nun denfolben îhagnet in einer Entfernung von i, 5 Fuls vom Meridian der $\mathrm{Na}$ del, mit feinem Südpole dem Nordpole der Nadel, an der weftliclren Seite gegen über, damit die $\mathrm{Ab}$ weichung.durch die Auziehung vermehrt würde, erkälteten ihn dann bis $0^{\circ}$ durch Schnee, und er. hitzten ibn wieder durch heifses Waller bis $+80^{\circ} \mathrm{C}$., 


\section{[ 291$]$}

Flüfigkeit der Ercle durch Vertheilung, indem fie alle'auf der Erdoberfläche' zerftreute magnetifche Flüfliglieit nach cler von der Sonne abgewandten Seite zurück ftülst, und die Magnetnadel ftrebe, nun nach den Orten $z u$, wo diefe irdifche magnetifche Flüflgkeit in Menge angehäuft fey. ${ }^{*}$ )

und fanden, dafs bei diefer Erwärmung die Abweichung fich um $5^{\prime} 46^{\prime \prime}, 5$ verminderte, welches für jeden Grad des Celfius' Tohen Thermometers 4",3 giebt. Es war alfo durch Erwärmung nicht blols die abftolsende, Iondern auch die anziehendo Kraft des Mlagnets verringert worden. Éndlich wurde derfelbe Magnet der Nadel bis auf 0,9 Fuls genähert, und die Declination erff bei einer $\mathrm{T} e m$. peratur des Mlagnets ron $+70^{\circ} \mathrm{C}$, hernach bei $0^{\circ}$ beobachtet. Die magnetifche Kraft war jetzt fu gewachfen, dafs die Declination durch diefe Erwärmung um einen Winkel von $12^{\prime}, 0^{\prime \prime}, 9$, oder für jeden Cellius'fchen Thermometergrad un 10", 3 z,ugenommen batte. Daraus fchliefsen wir, dafs dio Intenfität der Anziehungs- und Abftofsungskraft des Magnets durch die Wäme vermindert, durch die Kalte vermehrt wird, wenn fie auch nach horizontaler und mit dem magnetifchen Meridian normaler Richtung wirkt. Die Nadel war bei diefen Verfuchen in einer glafernen Kapfel eingefchloflen, und wir glauben daher, dals ihre Temperatur unverändert geblieben fey. Die Verfuche wurden in wenig Minuten gemacht, damit wir ficher waren, dafs die beobachreten Veranderungen einzig dem Magnete bsizume $\iint_{e n}$ feyen. Hällftr.

*) Mémoires de Mathém. et Phyf. preff. à l'Acad. roy.. Paris 1780 , Tom. IX, p. $262 . \quad$ Hïllitr. 


\section{[ 292$]$}

Anderer Schwierigkeiten nicht zu gedenken, glauben wir Canton's, viellejcht auch Preroft's und Coulomb's Hypothefe durch folgende Schlüffe und Erfahrungen umftofsen zu können.

Es ftelle der Kreis HOSW, (Fig. I, Taf. V,) den fcheinbaren Horizont des Beobachters, der in $C$ fteht, vor; $N S$ den wahren, $A B$ den magnetifchen Mericlian des Ortes; und der Winkel $N C A$ die Abweichung der Magnetnadel. Ift ferner $W C O$ fenkrecht auf $N S$ gezogen, fo giebt $O$ Often, $W$ Weften. Ebenfalls ziehe man durch $C, D C E$ fenkrecht auf den magnetifchen Meridian $A B$. Wenn nun die Sonne Morgens in der Verticalebene ift, welche durch $C$ und $\boldsymbol{E}$ geht, fo dals der öftliche von $N S$ liegende Theil der Erde erwärmt wird, fo mufs, nach $\mathrm{Ca}$. ton's. Hypothele, der Theil $A W B$ grölsere magnetifche Kraft haben, als $A E B$; und, nach Coulo $\mathrm{mb}$, das magnetifche Fluidum der Erde von $A E B$ nach $A W B$ getrieben werden, wodurch die Nadel ftärker nach $A W B$, als nach $A E B$ bewegt wird. Folglich muts alio derivlittelpunkt der Anziehung in der Verticalebene $C D$ feyn, fo dafs eine in $C$ fchwebende Magnetnadel in iler Richtung $C D$ angezogen wird. $C D$ drucke diefe Anziehungskraft aus, welche einzig die Nadel vom Mleridian $A B$ gegen Weften abweishen machi. , Eben fo wird gefunden, dafs, wenn die Sonne in $O$.oder in der Ebene $\mathrm{CO}$ ift, der Mittelpunkf der Anziehung in $C W$, und die anziehende Kraft $=C W$ feyn nü̈fe. Da aber die Richtung diefer Kraft nicht fenkrecht auf dem magnetifchen 


\section{[ 2.93 ]}

Mericlian $A B$ ift, fo mufs fie in die beiden Kräfte $W F$, parallel mit $A B$, und $C F$, derfelben normal, zerlegt werden, und dann wird gefunden, dafs $C F$ die Kraft fey, welche die Nadel gegen Weften abweichen macht. Ift die Sonne in $G$, fo ift $C K$ diefe Kraft, welche die Abweichung vermehrt. Ift die Sonne in $B$, fo ift die Kraft, welche die Declination vermehren follte, $=$ o. Kommt fie weiter, nach $S$, fo ift die auf die Nadel wirkende liraft $C L$ gegen Often gerichtet. Eben fo, wenn die Sonne in $M$ ift, die Kraft $C R$; u. f. w. Hieraus erhellet deutlich, dafs, nach der Meinung Canton's und Coulomb's, die weftliche Abweichung an griilsten feyn müfe, wenn die Sonne in $E$ fteht, das ift, ungefähr un 5 Uhr Morgens, wenn die nöri-

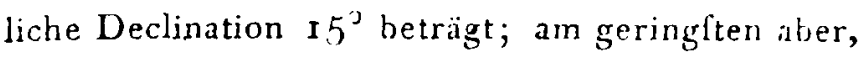
wenn die Sonne in $D$ fteht, das ift; um $5 \mathrm{Lhr}$ Nachmittags. Dies ftreitet aber gezen die Erfahrung, der zu Folge die weftliche Abweichung geraile Nachmittags um I, 2 oder 3 Uhr am ftärliften, Nachts aber an fchwachften ift. Diefes Wideripruchs wegen mülen wir die Hypothefen verwerfen.

Unter diejenigen, welche die Veränderungen der Abweichung aus den Magnete der Erde ableiten, find auch Macdonald, Aepinus und Hauy zu zählen. Sie nelmen mit Halley ${ }^{*}$ ) an, duils im Innern cler Erde ein Magnot verborgen liege,

*) Philof. Transact., Vol. XVII, n. 195.

H. 


\section{[ 294]}

deffen veränderte Kraft die Richtung der Magnetnadel beftinme. Macdonald *) fetzt voraus, dafs ein Pol nach den andern erwärmt und dadurch sn Kraft vermindert werde; ihn trifte claher das vorige Argument. A epinus und $\mathrm{Hauy}$ nehmen an, das magnetifche Fluidum fey im Magnete der Ercle ungleichförmig verbreitet, und daher rührten die Veränderungen der Abweichung. Wie fie indefs zu diefer Annahme berechtigt find, fehn wir nicht $a b$, und finden es auch nicht weiter erörtert. ${ }^{* *}$ )

Gegen die Hypothefen, welche einen in der Ercle befindlichen Magnet annehmen, macht $\mathbf{v}$ an Swinden ${ }^{* * *}$ ) die (ehr gegründete Einwendung, dafs diefe Erfcheinung nicht kosmifch fey, nicht von einer allgemeinen Urlache, die zu allen Zeiten und an allen Orten gleich wirke, abhänge. Denn aus den beigebrachten Beobachtungen erhellet, dafs diefe Veränderungen keinem Verhältniffe der Breite otler der Lānge dèr Orte folgen, bald an einem Orte zu einer Zeit grofs, in deffen Nachbarfchaft klein, und im Sommer faft allenthalben gröfser als im Winter find, und dafs fie in verfchiedenen Nadeln von

*) Annalen der Phyfik, B. 2, St. 1, S. $118 . \quad H$.

**) Darflellung der Theoric der Electricität und des Magnetismus nach den Grundrätzen des Hrn. A epinus, von Hauy, überfetzt ron Karl Murhard. Altenburg 1802, p. 264 . $H$.

** ). Mém. préjentés à l'Acadeınie, Paris 1780, pag. 335.

H. 


\section{[ 295$]$}

einem Peobachter verfchieden, und in keiner Uebereinftimmung mit der Inclination gefunden worden find. Wir können noch hinzu fügen, dais bei uns die weftliche Abweichung zuweilen Mittags am gröfsten, Nachts am kleinften, und zuweilen Nlittags am geringften und Nachts am grüfsten gefunden ift. Dielem allen zu Folge ftimmen wir mit van $S$ winden darin iberein, dafs diefe Erfcheinungen nicht aus dem Magnetismus der Erde zu erklären find.

Braun *) behauptet, die Schwingungen der Magnetnadel hingen von der Electricität der Luft ab; Cotte und Wilke ftimmen damit überein, und de la $C$ epè de ${ }^{* *}$ ) fagt felbft: Verfuche hätten gezeigt, dafs die electrifche Materie in der umgebenden Luft die Urfache der.Veränderungen feyn, und dafs diefe ganz aufhören, wenn die Nadel mit Nichtleitern umgeben werde. Er führt indefs nicht an, was das für Verfuche gewefen find, auch kennen wir fie nicht, wiffen aber, dafs eine mit Wachs überzogene Nadel die täglichen Veränderungen zeigt. Was aber auch für Gründe diefe Hypothefe für fich haben mag, fo ftreitet doch dagegen der, tafs die Veränderungen der Abweichung nicht immer grofs frnd, wenn die Intenfität der Luftelectricität vorzunglich ift, fo dafs es oft fcheint, als werde jene geringer, wenn diefe wächft, u. f. w.

*) Nov. Comm. Acad. Scient. Petrop.

H.

**) Lichtenberg's Mag., B. I, St. 1, S. 147. H. 


\section{[ 296 ]}

Da, fo viel Herr Hällftróm weils, noch niemand Verfuche uber den Einiuls der Electricität des die Vaclel umgebenden Nittels, auf ihre Abweichung, odler eines Magnets auf feinen Magnetis: mus angeftellt hat, lo ua:turndlun cr folgende Verfurlie, die ich hior mit binen liorten herfetze: "Wir tauchten eime horizont:ile, froi an einem FadeII voll Menfchenharen alligelsüs; is: Niddel in ein glalernes Gefäfs roll Wialier, wilil :ielıteten alles fo cin, dals clie Veränderungen der il iweichung durch das Mlikrolkiop bis atif wenijiftens $15^{\circ \prime \prime}$ bemerkt werden konṇtev. Durch einen melfingenen Draht führten wir dem Waffer aus einer Electritirmafoline Electricität zu, und beouachteten clabci die Naclel, konnten aber nichlit die misulefte Aesulerung in der Richtung derfelben enteclien. Wir verınochtea: diefes eben fo wenjg, wenn wir die Nadel felbft electrifirten; und es war lieine Aenderung fichtbar, die E.lectricität moc̣lıte lẹhwach, oder fo ft:ork feyn, dafs wir Funken aus dem Wafler ziehen konnten, verınieden wir pur., dafs die der Nadel inilgetheilte Electricität nicht durch Anzieben oder Abftotsen auf andere Kürper wirḳen, und dadurch die diaclel in ibrer Ricútung ftören! !onnte.

Wir legten nun eituen kiultichen Magnet, deffen Sudpol vom Nordpole der . Nignetuadel $g$ fchwedifche Zoil alstand, auf eine Gisstafel, und nach. den die iallel zur Ruhe gekommen war, theilten wir ihn Elodiricität nit, während cieffen wir die Sadel beoidchteten. Es war aber keine Verände- 


\section{[ 297 ]}

rung in ihrer Richtung zu beobachten. Daffelbe war der Fall, als wir den Nordpol des Magnets dem Nordende der Nadel gegen über ftellten.

Der Erfolg diefer Verfuche, der fich iminer gleich war, die Electricität mochte pofitiv oder negativ feya, von einer Electrifirmafchine, oder aus einer Leidner Flafche herrühren, zeigt, dafs $\mathbf{v}$ a n Sivinden Recht hat, wenn er läugnet, dafs clie electrifche Kraft die Declination der Nadel vermehre, und dafs wir mit ihm den Grund der Veränderungen der Abweichung der Nadel in der Nadel felbft und nicht aufser ihr fucher mülfen. Er hatte gezeigt, dafs in gewiffen Fällen die Abweichung der Magnetnadel vermehrt oder vermindert werden müffe, wenn die Intenfität der magnetifchen Liraft des.einen oder des andern Poles wachle oder abnehme, und oft wiederhohlte Verfuche hatten ihu belehrt, dafs diefe Veränder: fo. abwechfelnd fey, dafs fie felbft in kleinen Zeitmomenten nicht beftändig bleibe. Er vermuthet daher, fie fey periodifch, wie die Declination der Magnetnadel. Die Urfachen davon konnte er nicht ergründen.

Aus allem diefem fcheint zu erhellen, dafs die Urlache der täglichen Veränderung der Abweichung der Magnetnadel noch nicht erklärt fey. 